\title{
Rheological study of self-compacting mortars based on ternary cements
}

\section{Etude rhéologique des mortiers autoplaçants à base des ciments ternaires}

\author{
Dada Elhadja ${ }^{1}$, Belaidi Akram Salah Eddine ${ }^{1}$, Soualhi Hamza ${ }^{2}$ and Kadri El-Hadj ${ }^{2}$ \\ ${ }^{1}$ Laboratoire de recherche en génie civil (LRGC), Université de Laghouat, Algérie \\ ${ }^{2}$ Laboratoire de mécanique et matériaux du génie civil, Université de Cergy-Pontoise, France.
}

\begin{abstract}
Self-compacting concrete (SCC) is able to provide the ability to be easily implemented without vibration and to achieve spectacular structures, by its high fluidity and its rheological stability. By against its formulation requires a large volume of cement, which is necessary to allow its flow. The current environmental considerations lead to reduce the production of clinker however, it is essential to use cementitious additions to replace cement, because of their high availability and their moderate price. Furthermore, their use contributes in a simple and economical way to solve the problems related to the environment. The objective of our work is to study the effects of the incorporation of mineral additions such as: blast furnace slag of El-Hadjar (BFS), and marble powder (MP) on the rheological parameters of selfcompacting mortars developed in different combinations in ternary system with a substitution rate ranging from $20 \%$ to $60 \%$. According to this study, it been found that the substitution of cement by blast furnace slag and marble powder has negatively affected the rheological behavior of the mixtures. In addition, a considerable decrease in the rheological parameters has been achieved with a substitution rate of $20 \%$ of slag and $30 \%$ of marble powder. As well as an improvement of workability has been proven to self-compacting mortars and this is due to the increase of ternary cement replacement rate by marble powder from $20 \%$ to $30 \%$.
\end{abstract}

Keywords : blast furnace slag ; marble powder; ternary mortars ; viscosity ; yield stress

Résumé. Le béton autoplaçant (BAP) est capable d'assurer les aptitudes à être aisément mis en œuvre sans vibration et de réaliser des structures spectaculaires, par sa fluidité élevée et sa stabilité rhéologique. Par contre sa formulation sollicite un grand volume de ciment qui est nécessaire pour permettre son écoulement. Les considérations environnementales actuelles incitent à réduire la production du clinker, en revanche il est indispensable d'utiliser des ajouts cimentaires en remplacement au ciment, du fait de leur grande disponibilité et de leur prix modéré. D'autre part, leur utilisation contribue de manière simple et économique à résoudre les problèmes liés à l'environnement. L'objectif de notre travail consiste en l'étude des effets de l'incorporation des ajouts minéraux tels que : le laitier de haut fourneau d'El- Hadjar (LHF), et la poudre de marbre (PM) sur les paramètres rhéologiques des mortiers autoplaçants élaborés selon différentes combinaisons en système ternaire avec un taux de substitution variant entre $20 \%$ à $60 \%$. D'après cette étude, on a constaté que la substitution du ciment par le laitier de haut fourneau et la poudre de marbre a affecté négativement le comportement rhéologique des mélanges. Par ailleurs, une diminution considérable des paramètres rhéologiques a été menue avec un taux de substitution de $20 \%$ de laitier et $30 \%$ de poudre de marbre. Ainsi qu'une amélioration d'ouvrabilité a été prouvée pour les mortiers autoplaçants ternaire et cela est dû à l'augmentation par la poudre de marbre de $20 \%$ à $30 \%$.

Mots-Clés : laitier de haut fourneau ; poudre de marbre ; mortier ternaire ; viscosité ; seuil de cisaillement.

\section{Introduction}

La rhéologie est définie comme étant la science qui traite l'écoulement, les déformations, et plus généralement la viscosité des matériaux sous l'action des contraintes. La rhéologie est capable d'intégrer l'étude de l'ensemble des substances, qu'elles soient liquides ou solides. L'intérêt de la rhéologie grandit chaque jour depuis l'apparition des nouveaux matériaux aux comportements complexes tels que: pâtes, addition, polymères, etc. Parmi ces matériaux les bétons autoplaçants, qui sont des bétons très fluides, qui se mettent en place et se serrent sous le seul effet de la gravité, Cependant, son ouvrabilité dépend fortement de son comportement rhéologique de la phase de mortier dont les propriétés sont dominantes. A cet effet, l'évaluation des propriétés rhéologiques du mortier autoplaçant est une partie intégrale de la formation des $\mathrm{BAP}$, et donc la connaissance des propriétés du mortier est utile [1].

Pour un souci à la fois écologique et économique, il est plus qu'important de formuler des mortiers autoplaçants (MAP) en se basant principalement sur des matériaux locaux et disponibles (naturels ou issus des 
déchets industriels) tels que : le laitier des hauts fourneaux et la poudre de marbre. Aujourd'hui, le développement des nouveaux matériaux intensifie le besoin de caractériser encore mieux son comportement et son écoulement. [3]. Les tests empiriques conventionnels ne suffisent plus à bien caractériser le comportement lors de l'écoulement et de la mise en place. Le développement de nouveaux appareils basés sur une approche plus fondamentale et rhéologique donne des résultats plus adéquats et reflète bien l'aptitude physique du matériau à se déplacer sous son propre poids. Actuellement le comportement rhéologique des bétons frai et mortier est considéré comme similaire à celui de fluide de Bingham qui est donné par :

$\tau=\tau_{0}+\mu \dot{\gamma}$

(1)

$\tau_{0}(\mathrm{~Pa})$ est le seuil de cisaillement de Bingham, décrivant la contrainte nécessaire pour commencer l'écoulement, $\mu$ (Pas) est la viscosité plastique de Bingham, qui est la résistance de la matière à s'écouler, et $\tau(\mathrm{Pa})$ et $\dot{\gamma}(1 / \mathrm{s})$ est la contrainte de cisaillement et le taux de cisaillement, respectivement.

Dans le cadre de ce travail de recherche, les changements rhéologiques des mortiers autoplaçants impliqués dans les cas des mélanges composés de ciment plus des ajouts, à savoir le laitier de haut fourneau, et la poudre de marbre en système ternaire a été évaluée par des essais d'ouvrabilité à l'aide de mini-cône et mini V-funnel et par des mesures des paramètres rhéologiques à l'aide d'un rhéomètre

\section{Plan expérimental}

Le ciment utilisé dans cette étude est de type CEMI 42,5. Les additions minérales utilisées sont le laitier de haut fourneaux (LHF) et la poudre de marbre (PM). Leurs propriétés chimique et physique sont présentées dans le Table 1. Afin de déterminer l'état de surface des additions minérales, une microscopie électronique à balayage (MEB) a été utilisée. La Fig. 1 (a), (b) montre la morphologie du laitier et de la poudre de marbre. Comme on le voit sur ces figures, le laitier présente une forme anguleuse avec une texture de surface rugueuse. Par contre la poudre de marbre présente une uniformité granulaire. Le type de superplastifiant utilisé à base de polycarboxylate de nouvelle génération (SP) avec un poids spécifique de $1,07 \mathrm{~g} / \mathrm{cm}^{3}$ et un extrait sec de $30 \%$. Pour le sable on a utilisé dans ce travail un sable alluvionnaire avec un poids spécifique et de coefficient d'absorption de l'eau $2,7 \mathrm{~g} / \mathrm{cm}^{3}$ et $0,9 \%$ respectivement.

Table. 1. Caractéristiques physiques et chimiques du ciment, du laitier et de la poudre de marbre.

\begin{tabular}{|c|c|c|c|}
\hline $\begin{array}{c}\text { Analyse } \\
\text { chimique } \\
(\%)\end{array}$ & $\begin{array}{c}\text { Ciment } \\
\text { (OPC) }\end{array}$ & $\begin{array}{c}\text { Laitier } \\
\text { (LHF) }\end{array}$ & $\begin{array}{c}\text { Poudre } \\
\text { de } \\
\text { marbre }\end{array}$ \\
\hline $\mathrm{SiO}_{2}$ & 21,6 & 36,60 & 0,42 \\
\hline $\mathrm{CaO}$ & 63,7 & 37,75 & 56,01 \\
\hline $\mathrm{MgO}$ & 1,6 & 3,77 & 0,12 \\
\hline $\mathrm{Al}_{2} \mathrm{O}_{3}$ & 4,00 & 6,38 & 0,13 \\
\hline $\mathrm{Fe}_{2} \mathrm{O}_{3}$ & 4,5 & 6,94 & 0,06 \\
\hline
\end{tabular}

\begin{tabular}{|c|c|c|c|}
\hline $\mathrm{SO}_{3}$ & 1,9 & 0,19 & 0,01 \\
\hline $\mathrm{MnO}$ & - & - & - \\
\hline $\mathrm{K}_{2} \mathrm{O}$ & - & - & 0,01 \\
\hline $\mathrm{T}_{\mathrm{i}} \mathrm{O}_{2}$ & - & - & 0,01 \\
\hline $\mathrm{Na}_{2} \mathrm{O}$ & 0,03 & - & 0,43 \\
\hline $\mathrm{Cl}$ & 0,018 & - & - \\
\hline Perte en & 0,3 & 8,37 & 42,8 \\
\hline Densité & 3,15 & 2,56 & 2,61 \\
\hline Finesse & 3150 & 4000 & 3600 \\
\hline
\end{tabular}

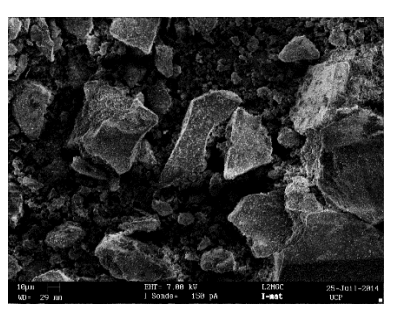

(a) Laitier de haut fourneau

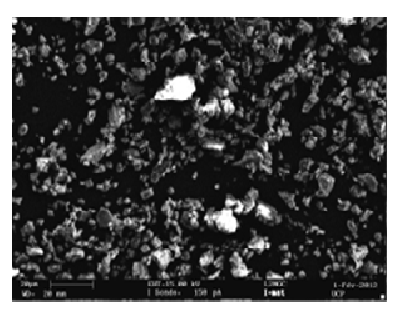

(b) Poudre de marbre
Fig. 1. Analyse par microscope électronique à balayage des additions

\section{Proportions du mélange}

Dans le BAP, la phase du mortier fournit la lubrification entre les particules des gros granulats et la stabilité globale du béton. Les propriétés du mortier sont semblables à celles du BAP lui-même, c'est-à-dire un faible seuil de cisaillement pour assurer l'écoulement sous le seul effet de la gravité et une viscosité plastique suffisante afin d'assurer la non ségrégation du béton durant l'écoulement. Toutefois, l'évaluation des propriétés des mortiers autoplaçant est une partie essentielle de la conception du BAP. Afin d'obtenir les propriétés recherchées à l'état frais du BAP, une proportion élevée de matière ultrafine et l'introduction des ajouts minéraux et chimiques sont nécessaires. Les proportions du mélange sont basées sur la méthode d'Okamura et Ozawa [4], avec des ajustements apportés à la méthode sur la quantité des granulats fins.

Les rapports en volume de sable sur mortier $\left(\mathrm{V}_{\mathrm{s}} / \mathrm{V}_{\mathrm{m}}\right)$, l'eau sur poudre $\left(\mathrm{V}_{\mathrm{w}} / \mathrm{V}_{\mathrm{p}}\right)$ et le superplastifiant sur poudre (SP/P) ont été déterminé par un simple test pour l'évaluation de la contrainte transférabilité du mortier frais [9]. Des essais sur mortier ont été réalisés pour le rapport Eau/poudre $(\mathrm{E} / \mathrm{P})$ constant de 0,4 en poids et le rapport sable / mortier $(\mathrm{S} / \mathrm{M})$ de 0,5 en volume. Les proportions de mélange de mortier de référence sont présentées dans le Table 2. LHF et PM ont été ajoutés comme une substitution de ciment en masse avec des pourcentages variant de $20 \%$ à $60 \%$ en système ternaire. Le Table 2 résume les formulations des mortiers ternaires.

La caractérisation rhéologique des mortiers a été effectuée avec un rhéomètre coaxial à palette. Ce rhéomètre a récemment été validé pour les mortiers et les bétons [14]. Les résultats bruts de l'essai sont les couples moment de cisaillement et les vitesses de rotation $\left(\mathrm{M}_{\mathrm{i}}\right.$, $\Omega i$. Pour exploiter les résultats en forme d'un rhéogramme de comportement rhéologique il faut passer par les équations (2) à (5) permettant de passer du couple 
$\left(\mathrm{M}_{\mathrm{i}}, \Omega_{\mathrm{i}}\right)$ au couple contrainte de cisaillement et taux de cisaillement $\left(\tau_{\mathrm{i}}, \gamma_{\mathrm{i}}\right)$ et de déterminer les paramètres rhéologiques, la viscosité et le seuil de cisaillement $(\mu$, $\tau_{0}$ ) du mortier testé.

$$
\dot{\gamma}=2 M \frac{\partial \Omega}{\partial M}
$$

$$
\begin{aligned}
& \frac{\partial \Omega}{\partial M} \cong \frac{\Omega_{j+1}-\Omega_{j-1}}{M_{j+1}-M_{j-1}} \\
& \tau_{j}(M) \cong \frac{M_{j}}{2 \pi h R_{b}^{2}} \\
& \tau_{i}=\frac{1}{2}\left(\tau_{j-1}+\tau_{j+1}\right)
\end{aligned}
$$

\begin{tabular}{|c|c|c|c|c|c|c|c|c|c|c|c|c|c|}
\hline & Mélange & MAP 0 & MAP 1 & MAP 2 & MAP 3 & MAP 4 & MAP 5 & MAP 6 & MAP 7 & MAP 8 & MAP 9 & Sable & SP \\
\hline \multirow{3}{*}{ 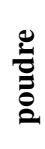 } & Ciments (\%) & $100 \%$ & $80 \%$ & $70 \%$ & $60 \%$ & $70 \%$ & $60 \%$ & $\mathbf{5 0 \%}$ & $60 \%$ & $50 \%$ & $40 \%$ & \multirow{7}{*}{2045.3} & \multirow{7}{*}{$1 \%$} \\
\hline & Laitier HF (\%) & $0 \%$ & $10 \%$ & $10 \%$ & $10 \%$ & $20 \%$ & $20 \%$ & $20 \%$ & $30 \%$ & $30 \%$ & $30 \%$ & & \\
\hline & Poudre de marbre $(\%)$ & $0 \%$ & $10 \%$ & $20 \%$ & $30 \%$ & $10 \%$ & $20 \%$ & $30 \%$ & $10 \%$ & $20 \%$ & $30 \%$ & & \\
\hline \multirow{4}{*}{ 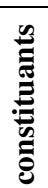 } & Ciments en $(\mathrm{g})$ & 1038.41 & 817.36 & 709.95 & 603.96 & 710.05 & 603.76 & 499.01 & 603.27 & 498.6 & 395.7 & & \\
\hline & Laitier en (g) & 0 & 102.17 & 101.42 & 100.66 & 202.87 & 201.25 & 199.6 & 301.64 & 299.16 & 296.77 & & \\
\hline & Poudre de marbre $(\mathrm{g})$ & 0 & 102.17 & 202.84 & 301.98 & 101.44 & 201.25 & 209.4 & 100.55 & 199.44 & 296.77 & & \\
\hline & Eau $(g)$ & 426.20 & 420.35 & 417.27 & 414.14 & 416.64 & 413.66 & 410.63 & 413.34 & 410.3 & 407.04 & & \\
\hline
\end{tabular}

Table. 2. Formulation des mortiers à base des ciments ternaires

\section{Résultats et discussions}

Les figures (2-a), (2-b) et (2-c) présentent l'effet combiné de laitier de haut fourneau et de la poudre de marbre sur le comportement rhéologique et la variation des paramètres rhéologiques des mortiers autoplaçants. On remarque que, au-delà de $10 \%$ de substitution par chaque addition, le laitier de haut fourneau en plus de la poudre de marbre affectent légèrement l'ouvrabilité des mélanges composés de ciment ternaire avec une quantité de laitier non élevé.

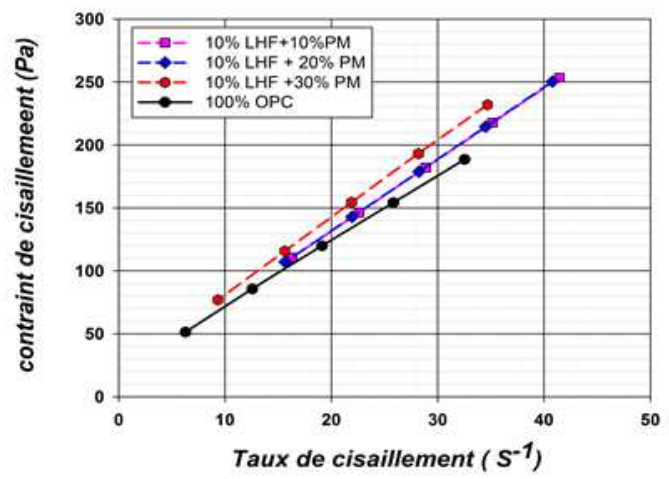

(a)

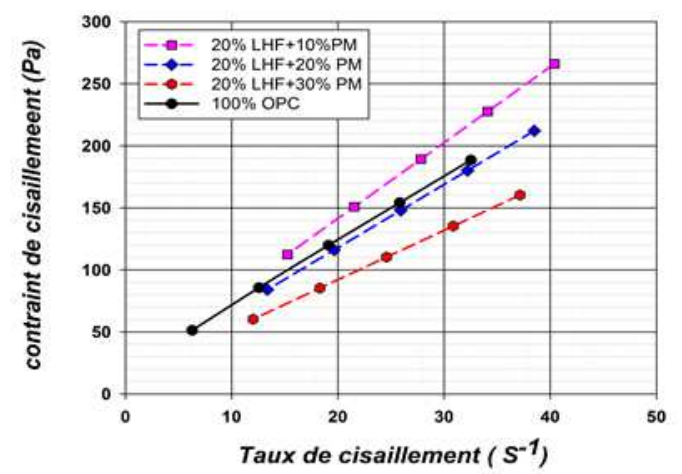

(b)

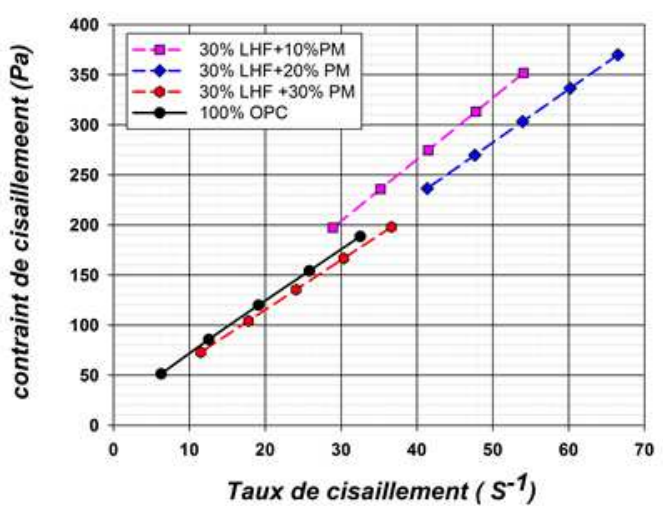

(c)

Fig. 2. Rhéogrammes des mortiers autoplaçants en fonction de dosage LHF et PM

Les figures (2-a et 2-c) indiquent une faible augmentation de viscosité qui est probablement négligeable. Notamment est dû à l'effet de dilution assuré par l'addition de la poudre de marbre de type inerte avec un taux d'incorporation de $20 \%$ à $30 \%$. De même, la figure (2-b) présente une diminution considérable de viscosité pour un taux de substitution de $20 \%$ LHF et $30 \%$ PM.

Parallèlement, les figures (3-a, 3-b et 3-c) si dessous présentent la variation de seuil de cisaillement et de la viscosité plastique des MAP en fonction du dosage en $\mathrm{LHF}+\mathrm{PM}$. D'où on remarque une diminution générale des paramètres rhéologique $\tau_{0}$ et $\mu$ ou delà de $10 \%$ de substitution de ciment par le LHF et de la PM par rapport au mortier de référence. 


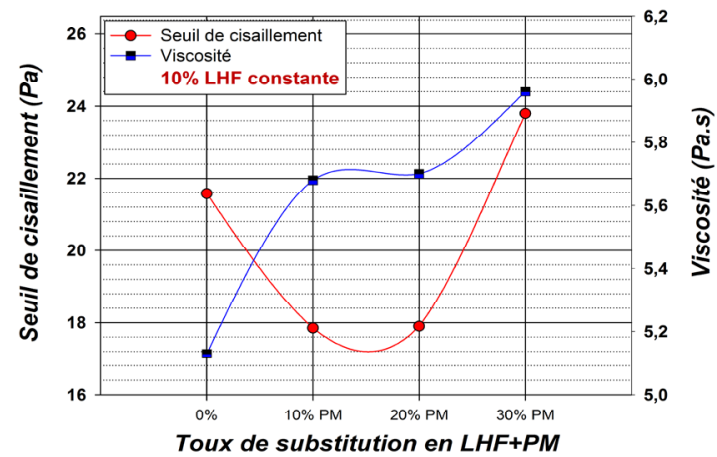

(a)

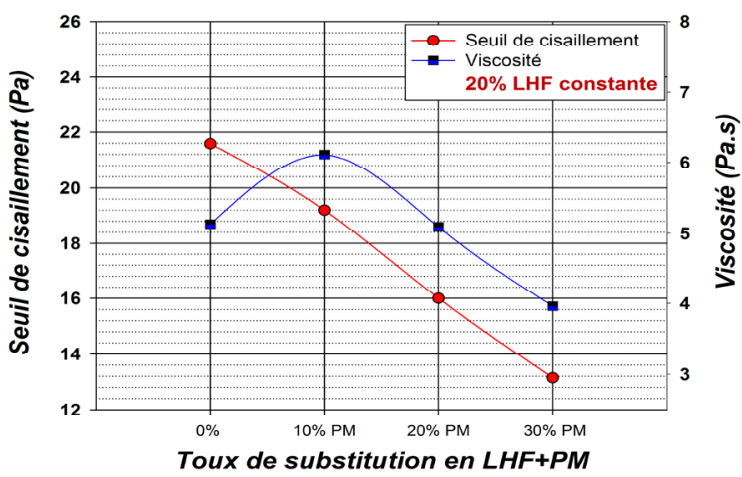

(b)

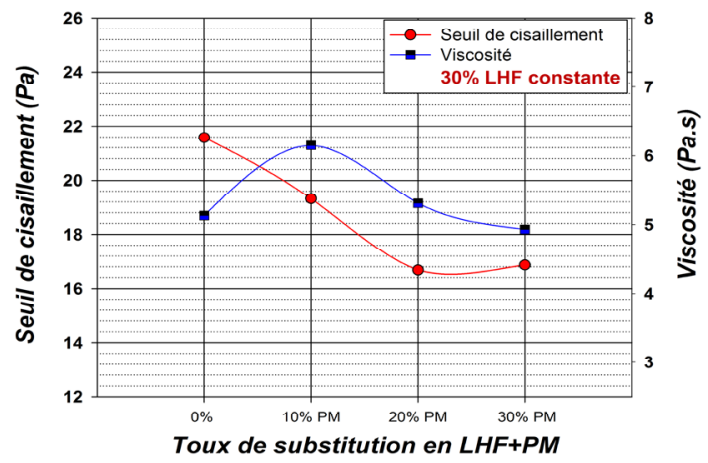

(c)

Fig. 3. Variation de seuil de cisaillement et de viscosité des MAP en fonction du dosage en LHF + PM

Les figures (4-a), (4-b) et (4-c) présentent les étalements et les temps d'écoulement obtenus avec le mini-cône et le mini v-funnel respectivement. On remarque que les essais l'ouvrabilité des MAP suivent la même tendance avec les résultats obtenu par le rhéomètre. On constate aussi que le remplacement du ciment avec un pourcentage de 20 à $30 \%$ de la poudre de marbre et un taux de $10 \%$ de laitier, on a une diminution dans l'étalement (figure 4-a) par rapport au mortier de référence, mais cette diminution reste dans l'intervalle d'exigence entre 27 et $33 \mathrm{~cm}$ [9].

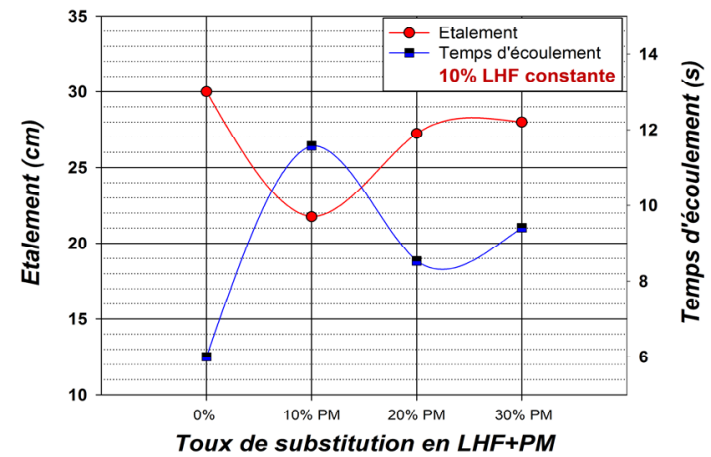

(a)

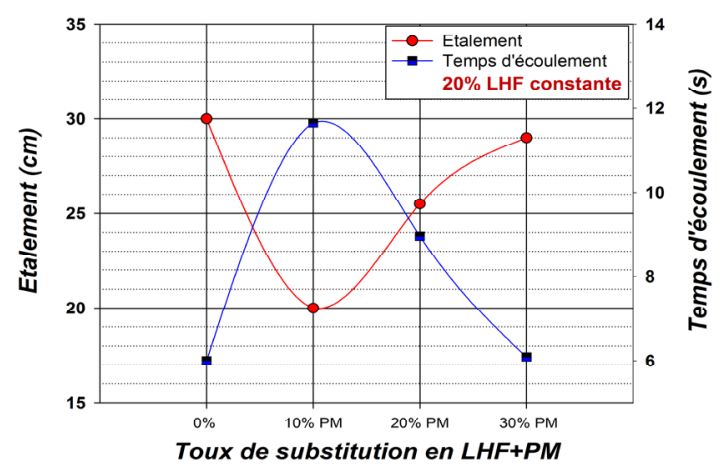

(b)

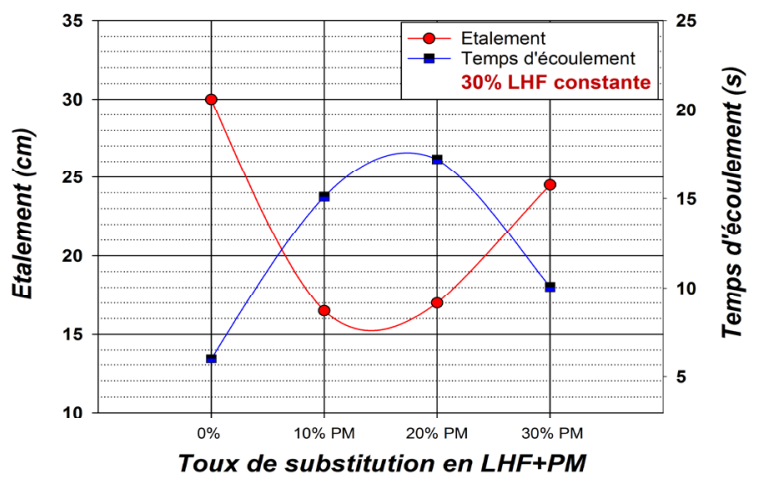

(c)

Fig. 4. Variation d'étalement et de temps d'écoulement des MAP en fonction du dosage en LHF + PM

Par conséquence, le temps d'écoulement par le mini vfunnel, on voit bien qu'on a une augmentation de temps de passage par rapport à celui de référence avec des temps entre 6,9 et $8,3 \mathrm{~s}$. Ces résultats restent toujours dans le domaine d'exigence fixé par Domone [9] entre 2 et $10 \mathrm{~s}$. Cependant, la combinaison ternaire de $20 \%$ LHF et 30\% PM indiqué dans la figure (4-b) présente un comportement comparable à celui de mortier de référence. Ainsi que la substitution de ciment par 10 à $20 \%$ de la poudre de marbre et un taux de $30 \%$ de laitier on à une augmentation de temps d'écoulement de 15 à 17 s figure (4-c) Probablement dû à la grande surface spécifique de laitier par rapport au ciment, qui nécessite une demande d'eau supplémentaire. 


\section{5 conclusion}

Les objectifs fixés dans cette étude étaient de montrer l'influence de la combinaison de laitier de haut fourneau et de la poudre de marbre sur l'ouvrabilité et les paramètres rhéologiques des mortiers autoplaçants à base des ciments ternaires. A la fin de cette étude on peut conclure les points suivants :

- L'utilisation du laitier de haut fourneau combiné avec la poudre de marbre dans les mortiers autoplaçants n'affectent généralement pas le comportement rhéologique et la maniabilité de mélanges à l'exception de quelques compositions ou l'effet de dilution par la poudre de marbre est clairement visible.

- La substitution de ciment par un dosage de 50\% d'ajout minéral (20\% LH et 30\% PM) est idéale pour un comportement rhéologique bénéfique, tout en respectant les exigences en vigueur.

- Ces résultats montrent la possibilité de fabriquer des mortiers autoplaçants à base de ciment ternaire avec un comportement rhéologique et une maniabilité adéquate par un simple ajustement au niveau du dosage en adjuvant.

\section{References}

1. A. S. E. Belaidi, «Contribution à l'étude rhéologique et durabilité de béton autoplaçant: apport des ajouts cimentaires ternaires et fillers locaux et industriels» thèse de doctorat, Université Saâd Dahleb de Blida, Algérie, (2013).

2. A. S. E. Belaidi, S. Kenai, H. Kadri, H. Soualhi and B. Benchâa, «Effects of experimental ternary cements on fresh and hardened properties of self-compacting concretes », Journal of adhesion science and Technology, vol. 30, no. 3, 247-261, (2016).

3. M. Adjoudj, «Effet des additions minérales et organiques sur le comportement rhéologique du béton », thèse de doctorat, Université Hassiba Ben Bouali de Chlef, Algerie, (2015).

4. H. Okamura and K. Ozawa, «Mix-design for self-compacting concrete », Concrete library of Jsce, no. 25, pp. 107-20, (1995).

5. Park and Noh, «Rheological properties of cementitious materials containing meneral admixture » Cement and concrete research, vol. 35, pp. 842-849, (2005).

6. M. Uysol and k Yilmaz, «Effect of mineral admixtures on properties of self-compacting concrete » Cement and concrete composites, vol. 33, pp. 771-776, (2011).

7. H. Okamura and M. Ouchi, «Self-compacting concrete », Journal of advanced concrete technology, vol. 1, no. 1, pp. 5-15, (2003).

8. A. S. E. Belaidi, L. Azzouz, E. Kadri, and S. Kenai, «Effect of natural pozzolana and marble powder on the properties of self-compacting concrete », Construction and Building Materials, vol. 31, pp. 251-257, (2012).

9. P. Domone and J. Jin, «Properties motar for selfcompacting concrete $\gg$ Procceding international symposium of self-compacting concrete, $\mathrm{pp}$. 109-20, Sep. (1999).

10. P. Domone, «Self-compacting concret: an analysis of 11 years case studies » Cement and concrete composites, vol. 2, no. 28, pp. 197208, (2006).

11. J. Golaszewski, «Influence of superplasticizers on rheological behaviour of fresh cement mortars », Cement and concrete research. 34 235-248, (2003).

12. C. Lanos, P. Estellé : «Vers une réelle rhéométrie adaptée aux bétons frais », Eur. J. Environ. Civ. Eng. 13, 457-471, (2009).

13. O. Taleb, F. Ghomari, M. A. Boukli Hacene, H. Kadri \& H. Soualhi, «Formulation and rheology of eco-self-compacting concrete (Eco-SCC)», Journal of Adhesion Science and Technology, Vol. 30, N³, 247-261, (2016).

14. F. De Larrard, «Concrete mixture proportioning a scientific approach», Modern Concrete Technology Series. No 9, Londres: E \& FN SPON ; (1999).

15. H. Soualhi, E.H. Kadri, T. Ngo, A. Bouvet, F. Cussigh, S. Kenai, «A Vane Rheometer for Fresh Mortar Development and Validation», Appl. Rheol, 24, 22594, (2014).

16. H. Chong, «Rhéologie des fluides », thèse de doctorat, Ecole Nationale des ponts chaussées, France, (2010).

17. P. Estellé, C. Lanos, A. Perrot, « Processing the Couette viscometry data using a Bingham approximation in shear rate calculation », J. Non. Newton. Fluid. Mech. 154, 31-38, (2008). 